\title{
An injectable nucleus pulposus cell-modified decellularized scaffold: biocompatible material for prevention of disc degeneration
}

\author{
Zhi Shan ${ }^{1, *}$, Xianfeng Lin ${ }^{1, *}$, Shengyu Wang ${ }^{1, *}$, Xuyang Zhang ${ }^{1, *}$, Yichuan Pang ${ }^{2}$, \\ Shengyun $\mathrm{Li}^{1}$, Tianming $\mathrm{Yu}^{1}$, Shunwu Fan ${ }^{1}$ and Fengdong Zhao ${ }^{1}$ \\ ${ }^{1}$ Department of Orthopaedic Surgery, Sir Run Run Shaw Hospital, Medical College of Zhejiang University, Hangzhou, 310016, China \\ ${ }^{2}$ MOE Key Laboratory of Macromolecular synthesis and Functionalization, Department of Polymer Science and Engineering, \\ Zhejiang University, Hangzhou, 310027, China \\ *These authors are contributed equally to this work \\ Correspondence to: Fengdong Zhao, email: zhaodong68@hotmail.com \\ Shunwu Fan, email: shunwu_fan@126.com
}

Keywords: intervertebral disc degeneration, small intestinal submucosa, nucleus pulposus cell, decellularization, extracellular matrix

Received: July 13, $2016 \quad$ Accepted: February 27, $2017 \quad$ Published: April 04, 2017

Copyright: Shan et al. This is an open-access article distributed under the terms of the Creative Commons Attribution License 3.0 (CC BY 3.0), which permits unrestricted use, distribution, and reproduction in any medium, provided the original author and source are credited.

\section{ABSTRACT}

We developed a nucleus pulposus cell (NPC)-modulated decellularized small intestinal submucosa (SIS) scaffold, and assessed the ability of this material to prevent Intervertebral disc degeneration (IVD) degeneration. Decellularized porcine SIS was squashed into particles and the biological safety and efficiency of these particles were evaluated. Next, SIS particles were seeded with rabbit NPCs, cultured for two months in vitro, decellularized again and suspended for intervertebral injection. We demonstrated that use of the decellularization protocol resulted in the removal of cellular components with maximal retention of extracellular matrix. The xenogeneic decellularized SIS did not display cytotoxicity in vitro and its application prevented NPC degradation. Furthermore, the xenogeneic SIS microparticles were effective in preventing IVD progression in vivo in a rabbit disc degeneration model. In conclusion, our study describes an optimized method for decellularized SIS preparation and demonstrated that the material is safe and effective for treating IVD degeneration.

\section{INTRODUCTION}

Low back pain is common in the elderly population, with a population prevalence of $15-30 \%$, and a lifetime prevalence of over $60 \%$. [1] Intervertebral disc degeneration (IDD) is believed to be the leading force of Low back pain by changing the biomechanical situation of the spine. [2] Also, the IDD can generate discogenic pain directly. [3] Low back pain associated with IDD can progress to multiple spinal disorders, including disc herniation, spondylolisthesis, and spinal stenosis. [4] Although there are many operative and non-operative approaches for treating IVD degeneration, such as pain control and discectomy of spinal fusion, most only address the symptoms and do not restore the natural structure of IVD.

Regeneration of degenerated disc tissues could restore IVD structure and treat IDD. While a proper extracellular matrix (ECM) could contribute to the regeneration of organs and tissues, decellularization is an attractive technique for preparing an ECM scaffold for tissue repair. Physical (shocks and freeze/thaw cycles), enzymatic (DNase and trypsin), and chemical (Triton X-100 and sodium dodecyl sulfate) protocols can achieve the decellularization of tissues and enable the acquisition of a cell-free ECM. [5] By removing the allogeneic or xenogeneic cellular components, the immunogenic response towards an ECM scaffold can be reduced while preserving most of the biomechanical and biological properties. Several studies have shown the potential of decellularization for the regeneration of IVD. Simionescu and $\mathrm{Xu}$ et $\mathrm{al}$. reported the decellularization of nativederived nucleus pulposus (NP) and annulus fibrosus (AF) tissues separately and demonstrated the biological compatibility of the material. [6-8] Cheung et al. [9] 
decellularized a whole IVD and removed $70 \%$ of the endogenous cells within the disc. Chan et al. considered collagen I to be the main component of NP ECM and obtained decellularized NP. [10] These studies show that decellularization is a feasible approach to obtain material for preparation of an ECM scaffold.

Decellularized NP has limitations when used as a scaffold, as the decellularization process can damage NP structure and bioactivity. [11] In addition, the NP is relatively difficult to acquire. SIS and NP share part of the ECM $[12,13]$, but when compared with NP materials, SIS is more economical, more convenient to acquire, and has a tougher structure. Thus, SIS could be an ideal scaffold material for regenerating IVD. As many researches supports that NP degeneration is a major part in disc degeneration process, the current study focused in retarding the NP degeneration [14]. Here, we used decellularized SIS (D-SIS) particles as a scaffold, used NPCs to modify the ECM of SIS, decellularized the SIS again (DD-SIS), and used the latter material to treat disc degeneration. A schematic outline of the study is depicted in Figure 1.

Porcine-derived SIS from small intestinal tissue applied as a completely decellularized matrix was first described as a vascular substitute. $[15,16]$ Research has shown that decellularized SIS contains bioactive factors such as fibronectin, glycosaminoglycan (GAG), and growth factors, [17] as well as bioactive factors involved in cell adhesion, mitogenesis, and chemotaxis. [18] Ligament reconstruction using SIS was demonstrated to be effective in equine, caprine, and canine models. [19-22]
Badylak et al. introduced SIS for Achilles tendon reconstruction in a dog model. [23] In a rodent model of abdominal wall defection, SIS displayed the ability to restore functional skeletal muscle structure. [24] Also, SIS has been proved to be useful in hernia repair, [25] Esophageal preservation, [26] skin replacement and wound repair in human. [27]

\section{RESULTS}

\section{Optimized decellularized method for SIS}

After the preparation of D-SIS and DD-SIS, H\&E staining was performed to evaluate the overall effect of the decellularization process. As presented in Figure 2A1 and 2A2, SIS presented pink eosinophilic staining indicating collagen. The effect of decellularization can also be confirmed by Masson staining (B1, B2). In contrast, no basophilic staining indicating nuclear material was observed in decellularized SIS (Figure 2A2). Alcian blue staining (Figure 2C1 and 2C2) showed that GAG content was partly preserved after decellularization to generate a suitable environment for NP cells. Immunohistochemical staining revealed that collagen type I and type II content was also preserved after decellularization. The degree of DNA decreased from $653.18 \mathrm{ng} / \mathrm{mg}$ before to $3.81 \mathrm{ng} /$ $\mathrm{mg}$ following decellularization. SEM analysis revealed complex fiber networks in the D-SIS, indicating that the SIS ultrastructure was maintained (Figure 3A). D-SIS particle size was approximately $200 \mu \mathrm{m}$ in diameter after 1st and 2nd time decellularization, a suitable size for cell

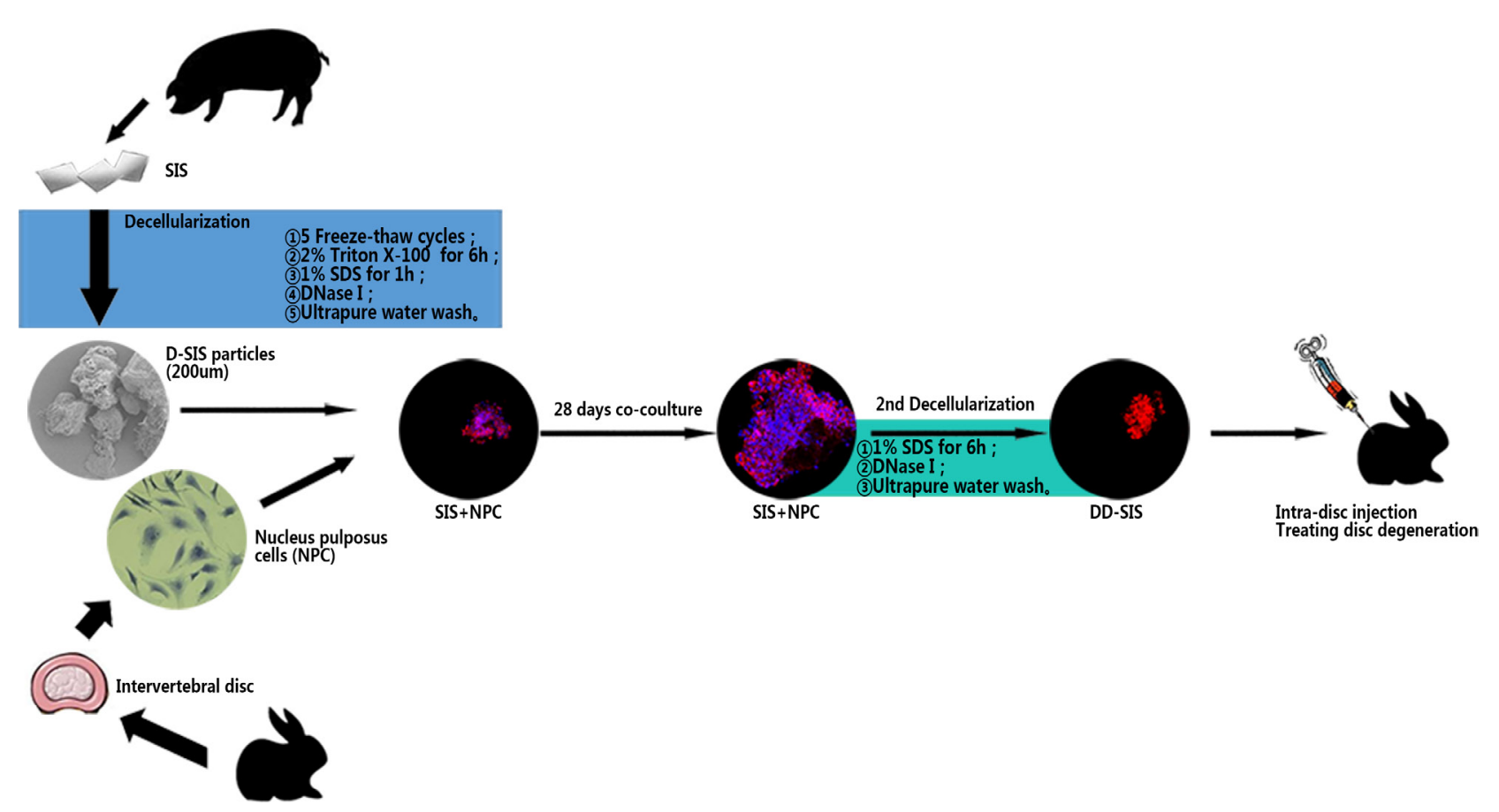

Figure 1: Schematic representation of the overall research. Abbreviations: SIS $=$ small intestinal submucosa; D-SIS $=$ decellularized SIS; DD-SIS = second decellularized SIS; NPC = nucleus pulposus cells. 
anchorage (Figure 3C). At the second decellularization, a milder protocol was used. H\&E staining, immunohistochemical staining, and DAPI staining showed complete removal of the cells, with preservation of the
ECM (Figure 4C4). Together, these data suggested that the decellularization strategy resulted in removal of the cellular components while preserving ECM content and structure.

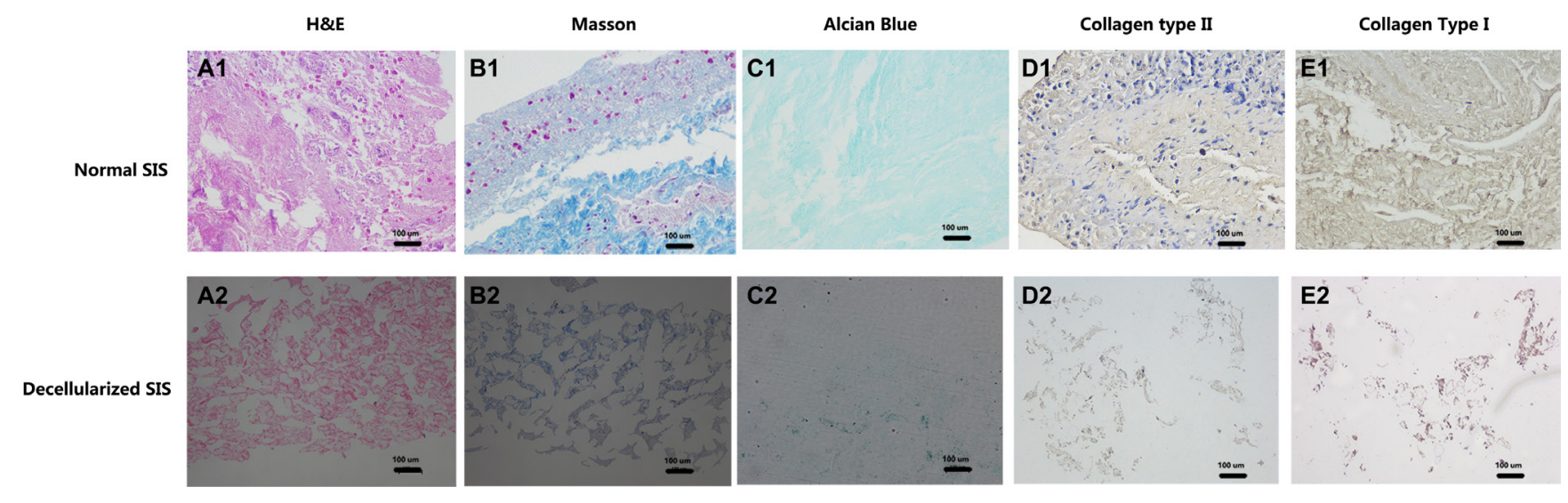

Figure 2: Effects of first time decellularization. Histological images of normal SIS (A1-E1) and decellularized SIS (A2-E2) confirmed the effect of decellularization, that no nuclear material can be found after decellularization, while part of collagen and aggrecan are maintained. Abbreviations: SIS = small intestinal submucosa.

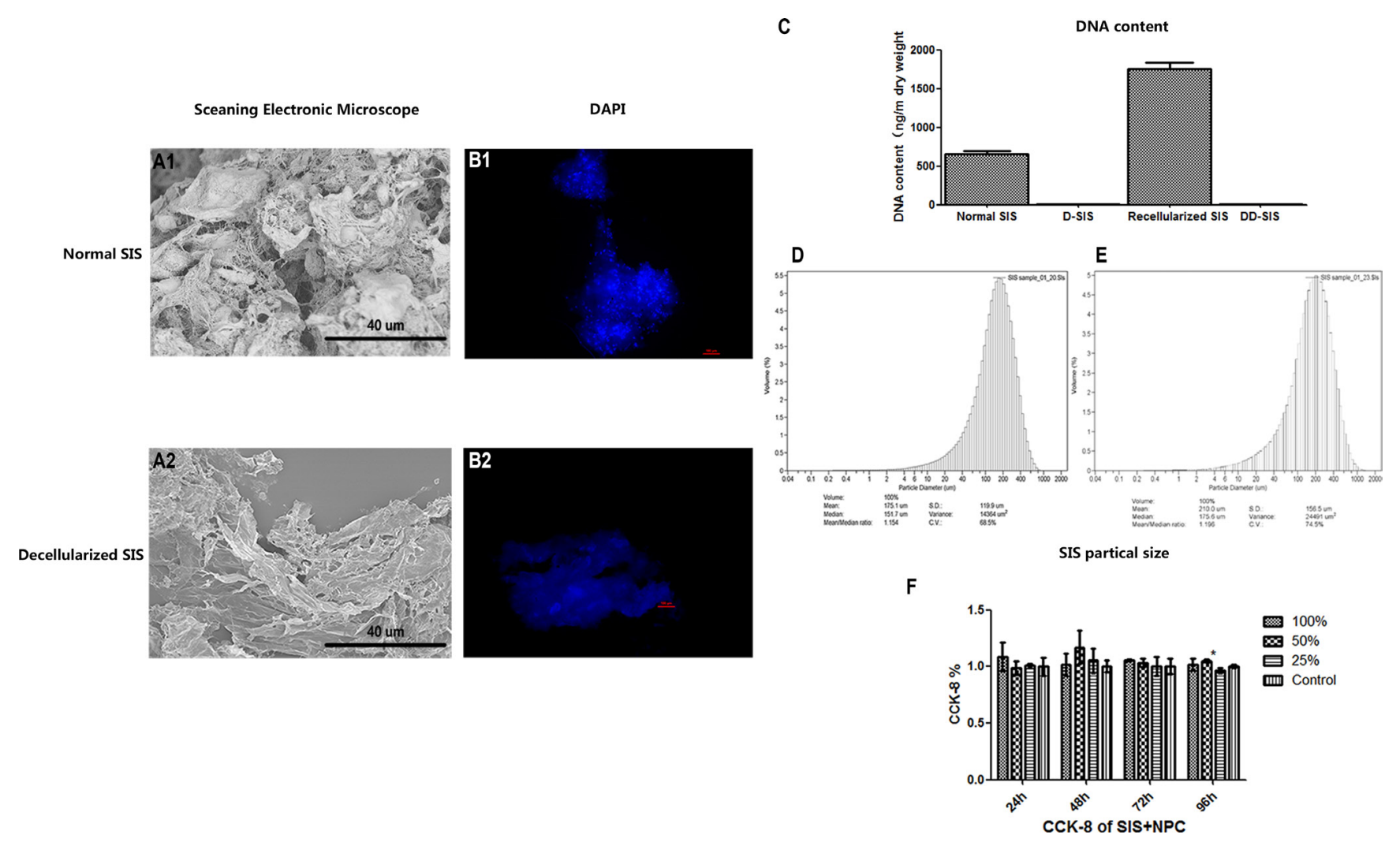

Figure 3: Effects of first time decellularization and SIS particle analysis. Scanning electronic microscope image showing that that the SIS ultrastructure was maintained (A), fluorescent images (B) and DNA content (C) of normal SIS, decellularized SIS, SIS recellularized with NPC and DD-SIS confirmed the efficiency of decellularization is good. SIS particle size was approximately $200 \mu \mathrm{m}$ after 1st (D) and 2nd (E) time decellularization. CCK-8 demonstrated good cytocompatibility (F). Results (C and F) are represented as mean +/- SEM. Abbreviations: SIS = small intestinal submucosa; DAPI = 4,6-diamidino-2-phenylindole, CCK-8 = cell counting kit-8. 


\section{Decellularized SIS supports NPC proliferation in vitro}

To modify the D-SIS to make it more suitable for IVD reconstruction, we repopulated NPCs onto the D-SIS suspension and evaluated the material in vitro. Live-Dead cell staining showed the long-term viability of NPCs after being seeded for 7, 14 and 28 days. At each time point, live cells (stained green) occupied the majority of the area (Figure 4A). As time progressed, more live cells were observed in the inner region of the D-SIS particles. H\&E staining confirmed that NPCs attached and proliferated with D-SIS (Figure 5A). DAPI staining also showed that cell distribution was in agreement with the above results (Figure 4B). Furthermore, we evaluated the morphology of these repopulated SIS using SEM, which revealed the presence of column, strip-like NPCs anchored on the SIS particles at day 14 . At day 28, cells maintained a striplike shape, which likely contributed to the maintenance of NPC morphological structure in vivo (Figure 4C).
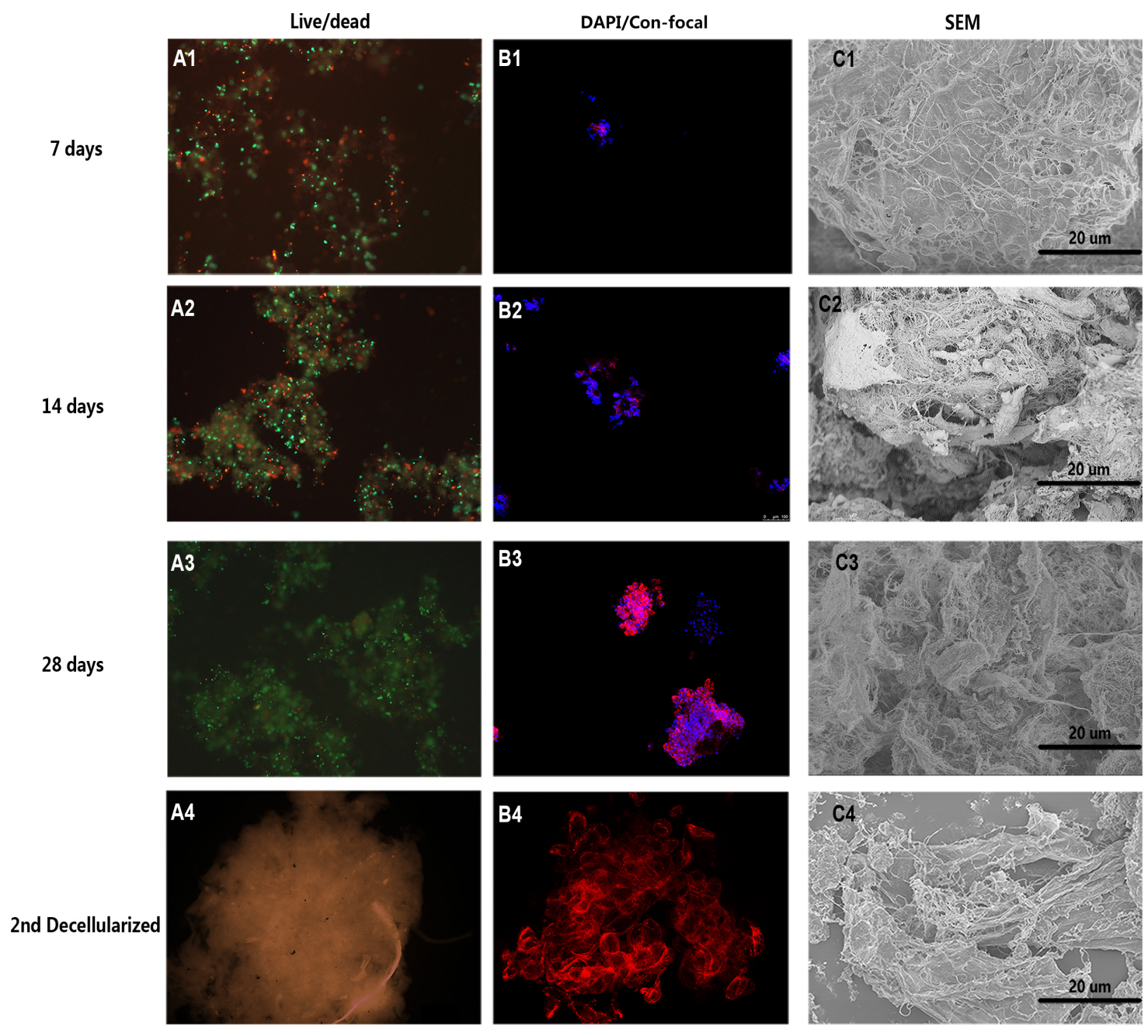

A5

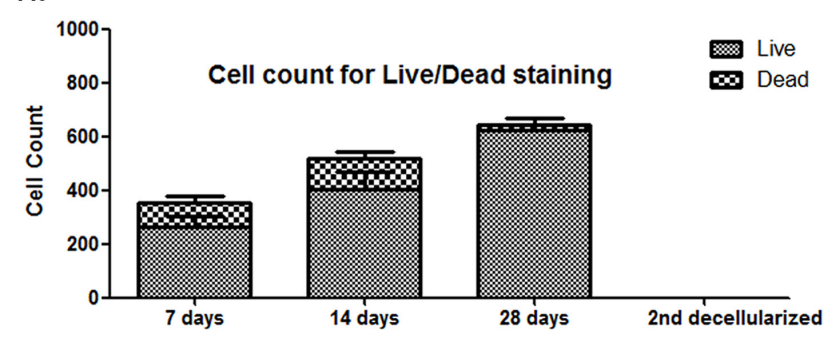

Figure 4: Decellularized SIS support NPC proliferation in vitro. Live-Dead cell staining (A1-A3), cell core confocal fluorescent images (B1-B3) and SEM (C1-C3) of NPC proliferation suggesting that decellularized SIS can support NPC proliferation generate ECM in vitro. After the second decellularization, all cell components were removed (A4-C4). The cell count for Live-Dead staining of each time point is shown in (A5). Abbreviations: SEM = small intestinal submucosa; DAPI = 4,6-diamidino-2-phenylindole. 


\section{Decellularized SIS supports NPC synthesis function in vitro}

To investigate the synthesis function of SIS repopulated with cells, we compared the expression of NP-related genes between NPCs seeded with D-SIS and NPCs cultured on a plate. RT-PCR results showed that when seeded in an SIS suspension, expression of genes encoding collagen type II, type I, and aggrecan increased after the first week and remained stable at day 21. Conversely, expression of these factors in NPCs cultured alone on a plate progressively decreased during the same period. At day 28, expression of aggrecan, collagen type II, keratin-18 and keratin-19 were 3.5-, 2-, 1.5- and 17fold higher than that of the control group, respectively.
Expression of the gene encoding SOX-9, a typical IVD cell marker, was 2.5 times greater than that in cells cultured alone. The ratio of collagen type II to collagen type I was also significantly greater in the SIS co-culture group. RT-PCR results are presented in Figure 6. In all, these results suggested D-SIS supported NPC survival and function in vitro.

\section{Decellularized xenogeneic DD-SIS prevented disc degeneration in vivo}

Given its ability to support NPCs in vitro, the effects of DD-SIS were further evaluated in vivo using a rabbit IDD model. Representative MRI and X-ray images from each time point are presented in Figure 7. The signal

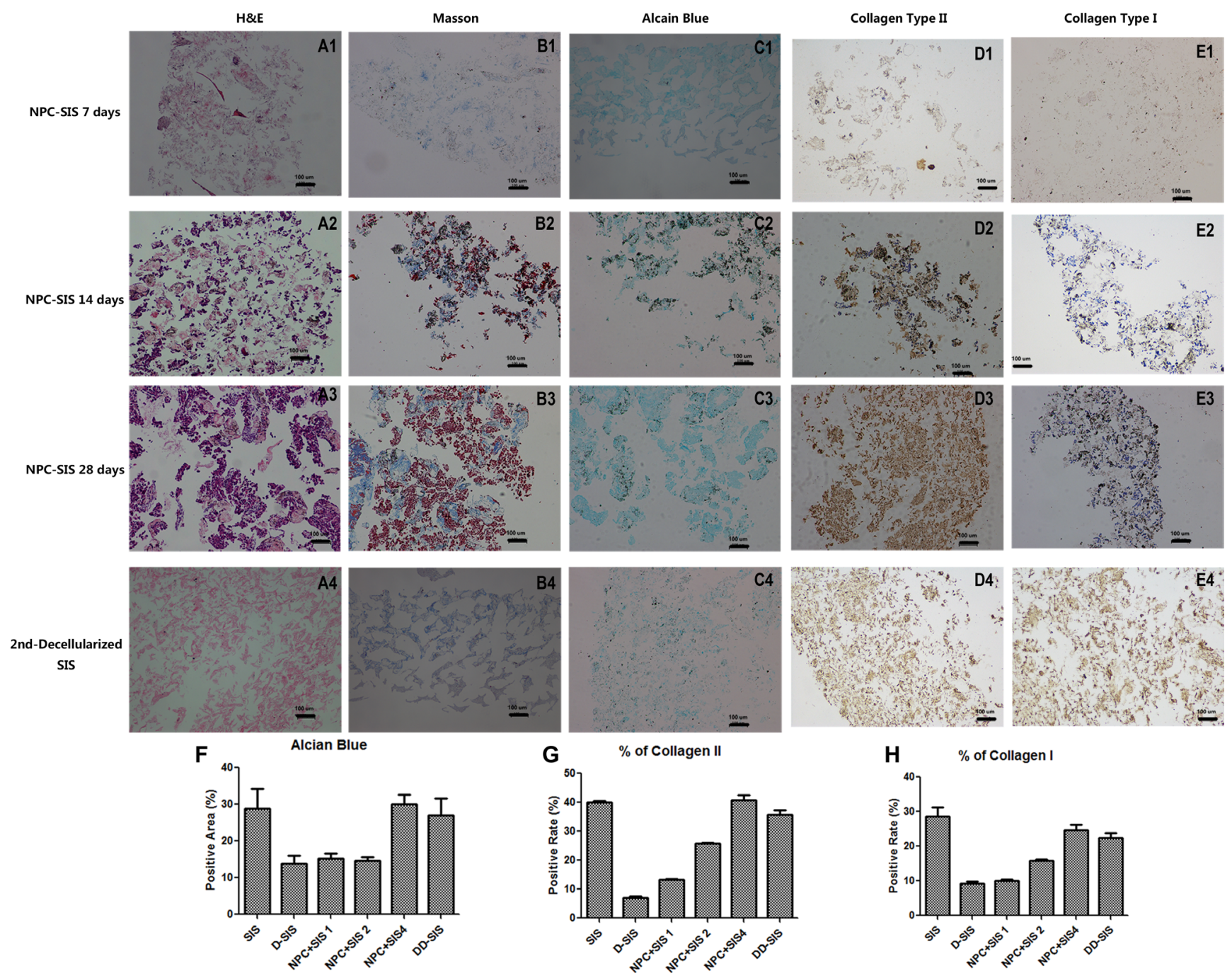

Figure 5: Decellularized SIS support NPC function in vitro. H\&E (A1-D1), Masson (A2-D2), Alcian blue (A3-D3), collagen type II (A4-D4) and collagen type I (A5-D5) staining suggesting that decellularized SIS can support NPC proliferation and generate ECM in vitro. After the second decellularization, all cell components were removed (E1-E5). Quantitative analysis showed suggesting that decellularized SIS can support NPC proliferation and generate Collagen II and GAG in vitro, and the second time of decellularization didn't damage the ECM $(\mathrm{F}-\mathrm{H})$. Results $(\mathbf{F}, \mathbf{G}$ and $\mathbf{H})$ are represented as mean $+/-$ SEM, and normalized to allow the exclusion of the blank areas. Abbreviations: SIS = small intestinal submucosa. 
intensity of IVD injected with saline showed a decreasing trend with time, while the IVD injected with DD-SIS displayed significantly higher signal intensity at 2 and 3 months $(P<0.001$, Figure 7A, 7B). When evaluated by $\mathrm{X}$-ray, disc height in the DD-SIS-treated group was larger than that in the saline-treated group, and the differences were significant between DD-SIS group and Saline/No treatment group at 2 and 3 months $(P<0.001$, Figure $7 \mathrm{C})$.

\section{DISCUSSION}

In this study, we developed an optimized decellularization method that could successfully remove the cellular components of SIS while maintaining its structure. NPCs grow well when seeded to the D-SIS in vitro and can modify the microenvironment, making the SIS more suitable for IVD reconstruction. We demonstrated that intradisc injection of DD-SIS has a therapeutic effect in an animal model of IDD.

SIS is one of the most established and broadly applied biological matrices. This type of matrix has been previously used for cardiovascular, [28] urogenital, [29] gastrointestinal [30] and ligament [23] reconstruction tests in vivo. Yang et al. observed that SIS can stimulate bladder smooth muscle cells and human umbilical vein endothelial cells to attach, proliferate and migrate in vitro. [31] Li et al. showed that small molecular weight peptides released by SIS are biologically active with respect to recruitment of murine endothelial cells in vitro and in vivo. [32] Cellseeded SIS has also been investigated. Du et al. reported a successful tracheal reconstruction in rabbits using SIS seeded with a monolayer of mesenchymal stem cells. [33] In this study, we firstly attempted to use NPC-modified SIS for treating intervertebral disc degeneration.

Since immune responses can facilitate IVD, decellularization was an essential step in our protocol. Because SIS is a dense tissue, we applied chemical treatments after physical treatment (snap-freeze/thaw cycles) to loosen the SIS tissue and remove the cellular antigens. The main bioactive components required for IVD regeneration, such as aggrecan, COL-I, and COL-II were well preserved upon both first and second decellularization processes. In addition, DNase was used, as insufficient removal of DNA fragments is associated with a greater risk of a pro-inflammatory or immune responses. [34, 35] All the washing steps of decellularization were carried at a low temperature $\left(4^{\circ} \mathrm{C}\right)$ to inhibit proteases and maintain SIS ultrastructure for SEM visualization. Therefore, we
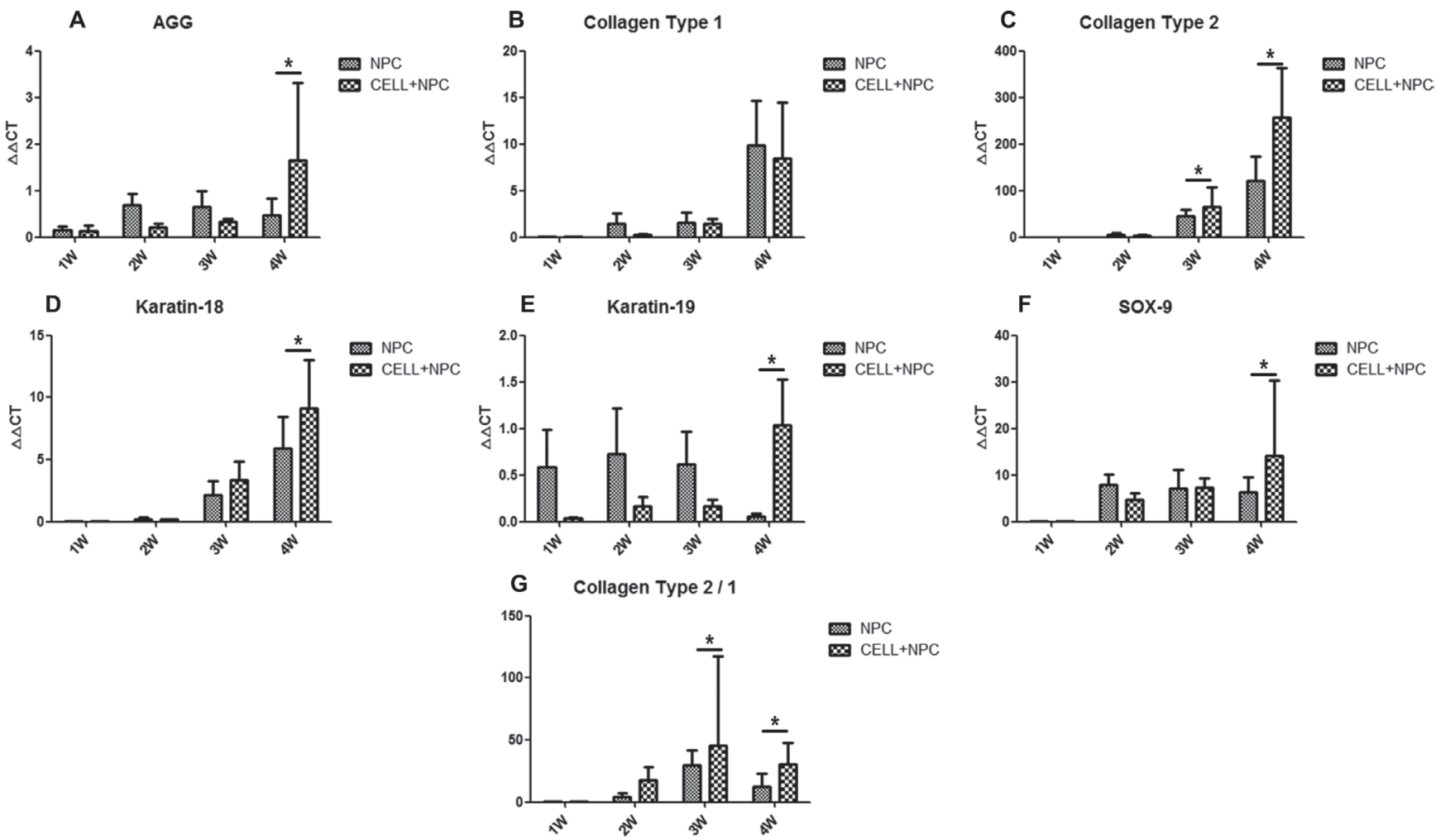

Figure 6: IVD-related gene expression in NPCs seeded in D-SIS evaluated by RT-PCR. Gene expression data are normalized to the average number of primary nucleus pulposus cells. Expression of aggrecan, collagen type I, collagen type II, keratin-18, keratin-19 and SOX-9 were significantly up-regulated at day 21 (A-F), the ratio of collagen type II/collagen type I was significantly greater in the SIS co-culture group $(\mathbf{G})$. Data are represented as mean +/- SEM. Abbreviations: AGG = aggrecan; COL-1 = collagen type I; COL2 = collagen type II. * indicates statistical significant difference. 
developed an optimized SIS decellularization protocol for IVD regeneration.

For intervertebral discs, there is still no effective screening platform for regenerative drug development. Cells are an indispensable part of tissue regeneration therapy. We showed that expression of functional NPspecific factors decreased when NPCs were cultured in a standard environment, suggesting potential spontaneous NPC degeneration. These results are consistent with previous reports showing that chondrocytes display decreased proliferation and matrix synthesis during expanded culture. [36] Therefore, scaffolds that can support normal NPC proliferation and synthesis are essential. By seeding NPCs into decellularized SIS, we observed that NPC degeneration was inhibited. With the support of SIS, NPCs maintained their original column and strip-like shapes, and their synthesis ability was preserved, as shown by the NP-related gene expression results. By binding to promoters of genes encoding Col-II and aggrecan, SOX-9 is also essential in NPC proliferation, $[37,38]$ and expression of these factors was preserved when NPCs were cultured with SIS. We conclude that a preserved collagen-rich microstructure, together with bioactive proteins and growth factors supported
T2

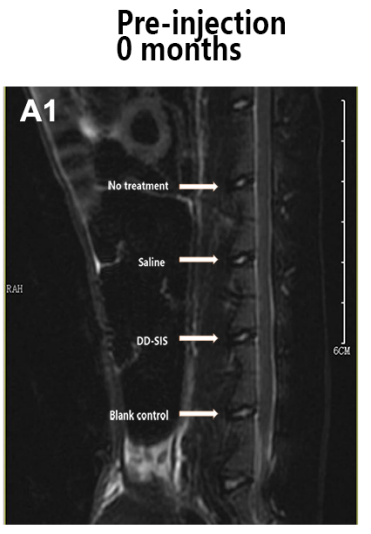

T1

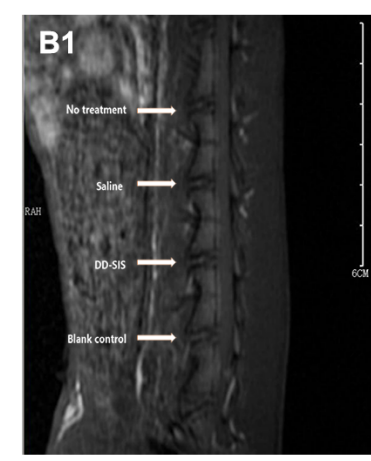

C

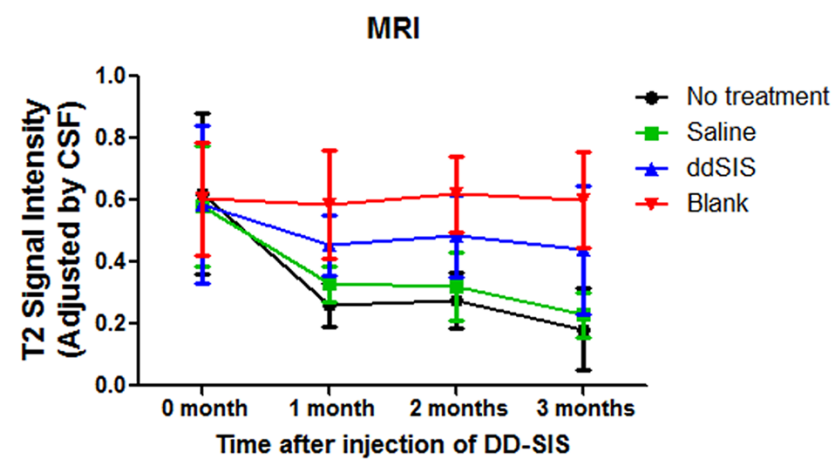

1 months
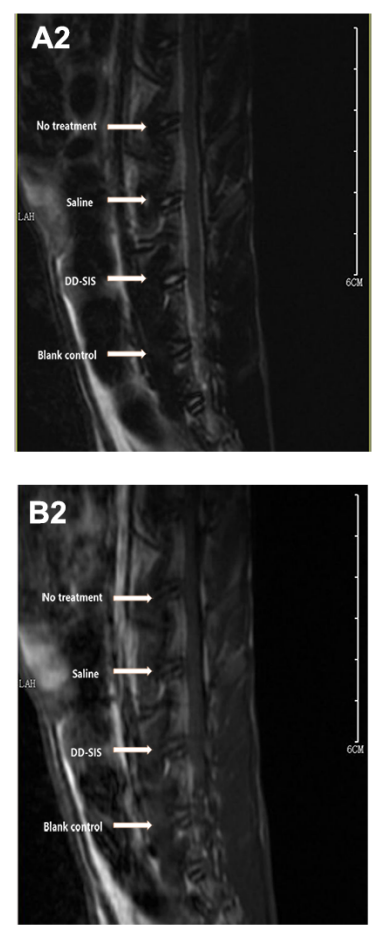

MRI
Post-injection

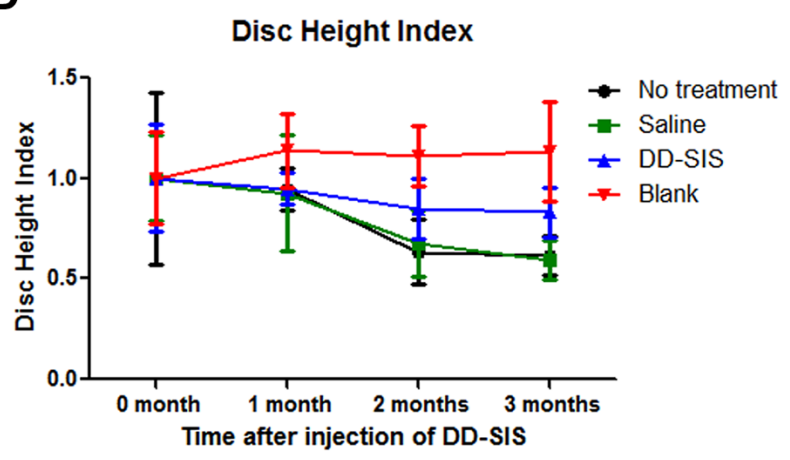

Post-injection

Post-injection 2 months
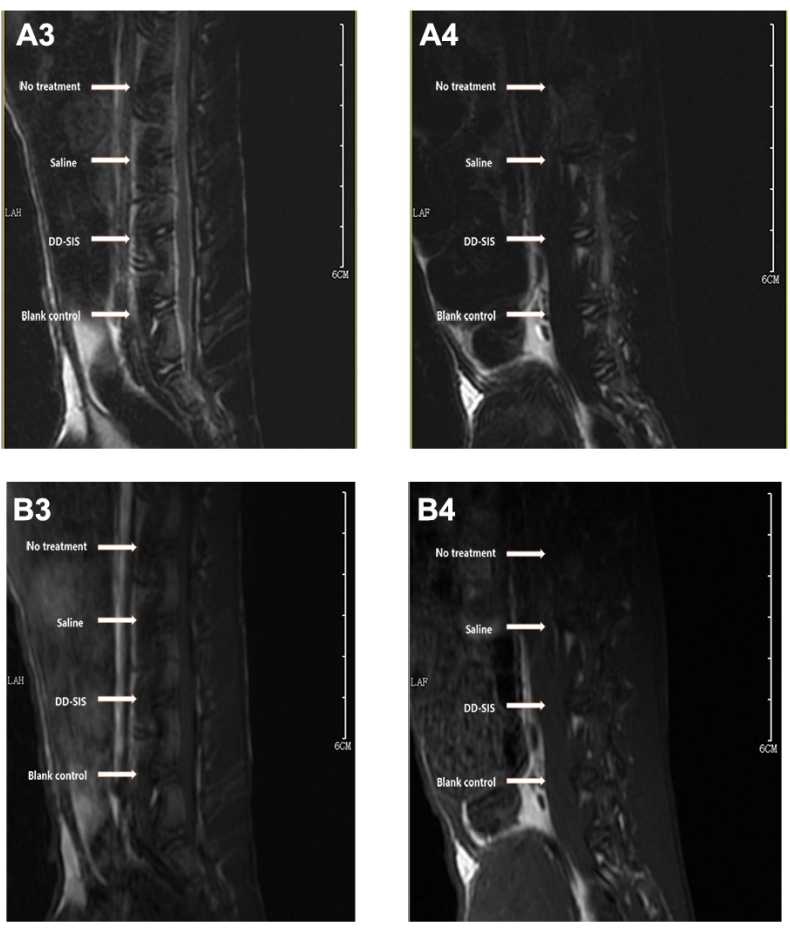

D

Figure 7: DD-SIS prevents disc degeneration in vivo. Representative continuous and dynamic changes of IVD revealed by magnetic resonance imaging (MRI) on T2-weighted (A) and T1-weighted (B) images. Quantitate analysis (C) of T2 disc signal intensity showed that DD-SIS prevents disc degeneration in vivo. Disc height index indicated by X-ray at different time points (D) showed better preserved disc height in DD-SIS group. $n=8$ per group per time point. Abbreviations: SIS $=$ small intestinal submucosa; DD-SIS $=$ second decellularized SIS. 
by the SIS, play an important role in inhibiting NPC degeneration. These factors, acted as footprints, facilitating NPC attachment, proliferation, and protein synthesis. In addition, a particle size of approximately $200 \mu \mathrm{m}$ was shown to be ideal for optimal cell adhesion [39, 40].

Being a widely-studied biomaterial, SIS has its technic and advantages. Also, the cost of NPC culturing and decellularization are acceptable. Thus, the economic feasibility of clinical usage of the DD-SIS is good. There were some limitations to our study. Firstly, we only tested a successful protocol for SIS decellularization and NPC culture. The effects of our protocols on different cell factors and signaling pathways associated with IVD regeneration were not explored in detail, and it requires further research. Previously articles have suggested that some growth factors, such as TGF- $\beta$, bone morphogenetic protein-2 and growth differentiation factor-5 could enhance the effect of intervertebral disc remodeling. [41-43] Secondly, we used injection of DD-SIS into discs of an animal model to treat IDD, while a needle puncture itself is an IDD risk factor. To reduce these negative effects, the needle size we used to inject DDSIS was $28 \mathrm{~g}$, which was much smaller than the size of the IVD. According to previous research, the punctureinduced degenerative changes can be largely affected by needle size. [44] Finally, a rabbit model with small IVD may be more favorable for regeneration, while this IVD degeneration model has been used in many researches and proved to be acceptable. Future research should focus on the mechanism of how SIS interacts with NPCs using proteomic and genetic approaches.

\section{MATERIALS AND METHODS}

\section{Research overview}

SIS samples were harvested from Bama miniature pigs aged two months and grinded into particles 100-300 $\mu \mathrm{m}$ in diameter. The cellular components were completely removed while the ECM structure of SIS was maintained. The determination of a successfully decellularized SIS was based on histological, DNA, and collagen content analyses. To evaluate the biological safety of xenogeneic SIS, the material was implanted into xenogeneic subcutaneous tissue in vivo. Rabbit NP cells were reseeded in decellularized SIS and cultured in vitro, and rabbit NPC growth was evaluated using histological, biochemical, real-time PCR, and electron microscopy. Next, after two months of co-culture, reseeded SIS was decellularized again, evaluated histologically, and injected in vivo to investigate its effects on treating of disc degeneration. Radiological evaluations of functional outcomes, such as disc height and degeneration level, were performed using X-ray and MRI analyses. All animal experiments were approved by the Animal Experimental Ethics Committee of Zhejiang University and animals were treated according to approved experimental protocols.

\section{Harvest and decellularization of SIS}

SIS material was collected from Bama miniature pigs that were approximately two months old. Jejunum sections were harvested within $2 \mathrm{~h}$ of sacrifice and then prepared according to a previously published method. [45] To separate SIS from porcine jejunum, fat was first removed, followed by careful washing with water and a saline solution, and then cut into pieces $10 \mathrm{~cm}$ in length. Tunica serosa and tunica muscularis were carefully removed mechanically using gauze. An optimized decellularized protocol should completely remove the cellular components of SIS while fully maintaining the three-dimensional structure of the ECM. Prior to other decellularization procedures, five snap-freeze-thaw cycles (liquid nitrogen and $37^{\circ} \mathrm{C}$ water bath) were performed to facilitate the diffusion of decellularized reagents. After freeze-thaw processing, SIS particles were decellularized using 2\% Triton X-100 (Sigma-Aldrich, St. Louis, MO, USA) for $6 \mathrm{~h}$ and SDS (1\%, Sigma-Aldrich) for $1 \mathrm{~h}$ and next processed with DNase I (100 U/mL, Sigma-Aldrich, $37^{\circ} \mathrm{C}, 6 \mathrm{~h}$ ). After decellularization, samples were washed in ultrapure water ( 2 days, 3 exchanges per day) and rinsed in PBS (1 day). All decellularization steps were performed by submerging the tissue in the decellularization reagents and incubating with agitation (120 rpm).

\section{Preparation of SIS particle scaffold}

The SIS pieces after decellularization were pulverized into 100-200 $\mu \mathrm{m}$ diameter particles with a tissue homogenizer (Precellys 24, Bertin Technologies, Paris, France) in liquid nitrogen (6,000 rpm, 20 cycles, 30 $\mathrm{s} / \mathrm{cycle})$. After pulverization, a suspension was obtained and filtered through an 80-mesh cell sieve $(200 \mu \mathrm{m})$, which controlled the size of harvested SIS. SIS particle sizes were tested using a Laser Particle Size Analyzer (LS13-320, Beckman Coulter, Brea, CA, USA). Next, SIS particles were immediately frozen in liquid nitrogen for later use.

\section{Isolation and culture of rabbit NP cells}

Rabbit NPCs were isolated from the NP tissue of two month-old New Zealand white rabbits with type I collagenase (5\%) and cultured in Dulbecco's modified Eagle medium with high glucose (DMEM-HG, Gibco, Grand Island, NY, USA) plus fetal bovine serum (10\%, Gibco, Mulgrave, Australia), penicillin (100 U/mL), streptomycin $\left(100 \mu \mathrm{g} \mathrm{mL}^{-1}\right)$, and amphotericin B $(2.5 \mu \mathrm{g}$ $\left.\mathrm{mL}^{-1}, 37^{\circ} \mathrm{C}\right)$ in a humidified atmosphere that contained $\mathrm{CO}_{2}(5 \%)$. Cells were seeded in T75 flasks and allowed to adhere for $24 \mathrm{~h}$. After three washes with PBS, adherent cells were cultured in complete DMEM-HG. Cells were detached using trypsin-EDTA (0.25\%) upon $80-90 \%$ confluence and subcultured at passage 1 (P1). Cells at P2 were used for the recellularization for the D-SIS. 


\section{Cytocompatibility testing of SIS (CCK-8)}

SIS cubes were incubated in DMEM-HG medium for $48 \mathrm{~h}$ in a humidified atmosphere with $\mathrm{CO}_{2}\left(5 \%, 37^{\circ} \mathrm{C}\right)$ at and the leaching solution was collected for later use. NPCs at P2 were seeded in 96-well cell culture plates (5 $\times 10^{3}$ cells/well $)$ in standard DMEM-HG $(200 \mathrm{~mL})$ for 24 h. The medium was then removed and replaced with the leaching solution $(25 \%, 50 \%$ and $100 \%)$ and incubated for six days. At days 1, 2, 3, and 4, cell counting kit-8 (CCK8) assay (Dojindo Laboratories, Kumamoto, Japan) was added to each well, and then the plate was incubated in the incubator (30 minutes).

\section{Repopulation of NP cells into an SIS particle scaffold}

Research has shown that appropriate ECM can delay spontaneous senescence of chondrocytes. [46] Therefore, we seeded NPCs $\left(7 \times 10^{6}\right.$ cells $\left.\mathrm{mL}^{-1}\right)$ into SIS particles suspended in DMEM-HG to evaluate its effect on degeneration. Cells from the same source were cultured with DMEM-HG $\left(5 \times 10^{5}\right.$ cells $\left.\mathrm{mL}^{-1}\right)$ as a negative control.

\section{Cell viability and metabolic activity of recellularized SIS}

After repopulation, a Live-Dead cell staining Kit (BioVision, Milpitas, CA, USA) was used to distinguish live and dead cells and the cellular activity of the recellularized SIS was observed at 1, 2, 3 and 4 weeks. Live NPCs (stained green) were visualized using fluorescence microscopy, counted and recorded as the mean of 10 randomly-selected views from each specimen at $10 \times 10$ magnification, and were compared with the positive control group (NPCs cultured in standard DMEMHG) to determine the cytocompatibility of SIS particles.

\section{Evaluation of histological, histochemical, immunohistochemical and fluorescent staining}

Normal SIS, D-SIS, recellularized SIS at 7, 14 and 28 days, and DD-SIS were washed with PBS three times, fixed in PFA (4\%, $24 \mathrm{~h})$, followed by standard paraffin embedding and sectioning. For in vivo treatment, the IVD were harvested from the rabbits three months after injection of DD-SIS, fixed in PFA $(4 \%, 48 \mathrm{~h})$, decalcified (30 days) and embedded in paraffin. Hematoxylin and eosin staining was performed to reveal the whole structure and cell distribution. Alcian blue staining was performed to evaluate retained GAG content. Specimens for immunohistochemistry were processed as previously described by our group. [47] Epitopes of interest included collagen type I (Abcam, Cambridge, MA, USA), collagen type II (Novus Biologicals, Littleton, CO, USA) and aggrecan (Novus Biologicals). 4,6-Diamidino- 2-phenylindole (DAPI, Sigma) fluorescent nuclear staining was performed to evaluate the efficiency of decellularization and NPC distribution at different time points.

\section{DNA concentration analysis}

Genomic DNA of SIS, D-SIS, recellularized SIS at different time points and DD-SIS was extracted and isolated using a DNeasy Blood \& Tissue Kit (Qiagen, Hilden, Germany), according to the manufacturer's protocol. DNA concentration was measured by $260 \mathrm{~nm}$ ultraviolet absorption using a NanoDrop 8000 instrument (Thermo Fisher Scientific, Wilmington, MA, USA).

\section{Scanning electron microscope}

Scanning electron microscopy (SEM) was performed to examine the microarchitecture of D-SIS and recellularized SIS. Particles were washed with $\mathrm{ddH}_{2} \mathrm{O}$, freeze-dried, sputter-coated with gold-palladium and viewed under the SEM (SU8010, Hitachi, Japan).

\section{Gene expression of IVD cell markers by RT-PCR}

The mRNA from NPCs-seeded SIS was extracted with RNeasy Mini Kit (Qiagen, Valencia, CA, USA) according to the manufacturer's instructions. RNA integrity and concentration were determined using a NanoDrop 8000 instrument. Reverse transcription was performed at $45^{\circ} \mathrm{C}$ for 50 minutes and $82^{\circ} \mathrm{C}$ for 5 minutes using $5 \times$ Prime Script RT Master Mix $(2 \mathrm{~mL}$, Takara Bio, Otsu, Japan), mRNA sample (1 $\mu \mathrm{g})$, and RNase-free $\mathrm{ddH}_{2} \mathrm{O}(4 \mathrm{~mL})$ in a total volume of $10 \mathrm{~mL}$. Reactions were set up in triplicate in 96 -well plates $(20 \mathrm{~mL}$ each well) using cDNA $(2 \mathrm{~mL})$ with SsoFast Eva Green Supermix (10 mL, Bio-Rad, Hercules, CA, USA), ddH $\mathrm{O}_{2}(7 \mathrm{~mL})$ and gene-specific forward and reverse PCR primers (10 $\mathrm{mM}$, Table 1). The expression of genes encoding collagen type I (COL-1), collagen type II (COL-2), SOX-9, aggrecan (AGN), keratin-18 and keratin-19 was quantified to investigate NPC growth. The housekeeping gene $G A P D H$ was used as a control for normalization, and the expression ratio for each marker of interest was determined using the $2^{-\Delta \Delta \mathrm{Ct}}$ method. RT-PCR reactions were performed at $95^{\circ} \mathrm{C}$ for $10 \mathrm{~min}$ (activation), followed by 40 cycles amplification $\left(95^{\circ} \mathrm{C}\right.$ for $10 \mathrm{~s}, 60^{\circ} \mathrm{C}$ for $20 \mathrm{~s}$, and $72^{\circ} \mathrm{C}$ for $20 \mathrm{~s}$ ), and a final extension $\left(72^{\circ} \mathrm{C}, 1 \mathrm{~min}\right)$, using an ABI Prism 7500 system (Applied Biosystems, Foster City, CA, USA).

\section{Second decellularization of SIS particles}

After reseeded D-SIS with NPC for 2 months, samples were again decellularized using SDS (1\%, Sigma) for $6 \mathrm{~h}$ and washed in ultrapure water $(12 \mathrm{~h})$. As with the 
Table 1: Nucleic acid sequences of forward and reverse PCR primers of specific genes

\begin{tabular}{|c|c|c|c|}
\hline Gene name & Species & Direction & Sequences \\
\hline GAPDH & Rabbit & Forward & 5'-ATGGTGAAGGTCGGAGTGAAC-3' \\
\hline GAPDH & Rabbit & Reverse & 5'-GTGGGTGGAATCATACTGGAAC-3' \\
\hline Collagen Type 1 & Rabbit & Forward & 5'-GAACGGAGATGACGGAGAAG-3' \\
\hline Collagen Type 1 & Rabbit & Reverse & 5'-TCCAAACCACTGAAACCTCTG-3' \\
\hline Collagen Type 2 & Rabbit & Forward & 5'-GCTCAAGTCCCTCAACAACC-3' \\
\hline Collagen Type 2 & Rabbit & Reverse & 5'-CCAGTAGTCACCGCTCTTCC-3' \\
\hline Sox 9 & Rabbit & Forward & 5'-GGGAAGCTCTGGAGACTGCT-3' \\
\hline Sox 9 & Rabbit & Reverse & 5'-TGTAGTCCGGGTGGTCTTTC-3' \\
\hline Keratin 18 & Rabbit & Forward & 5'-GCTGAAATCGCCACCTACC-3' \\
\hline Keratin 18 & Rabbit & Reverse & 5'-TGGTCTTCTGGATGGTCTGC-3' \\
\hline Keratin 19 & Rabbit & Forward & 5'-GCTGGCCTACCTGAAGAAGA-3' \\
\hline Keratin 19 & Rabbit & Reverse & 5'-GTCAATGCCTGGAGCAGAAT-3' \\
\hline Aggrecan & Rabbit & Forward & 5'-AACAGCCCAAGAAGCAGAAG-3' \\
\hline Aggrecan & Rabbit & Reverse & 5'-GGGTCCAGAAATCCAGAATG-3' \\
\hline
\end{tabular}

decellularization process (Chapter 5.3), samples were submerged in the decellularization reagent and incubated with agitation $(120 \mathrm{rpm})$.

\section{Establishment of an IVD degeneration rabbit model}

An IVD degeneration rabbit model was induced with a fine needle puncture. [48] The model was confirmed as valid based on previous research describing an MRI signal change within the disc and collapse of disc space. Rabbits were anesthetized with an intravenous injection of pentobarbital sodium $\left(15 \mathrm{mg} \mathrm{kg}^{-1}\right)$ and then positioned prone on a platform. Rabbit spines were exposed using a lateral retroperitoneal approach with aseptic techniques. L4-5 and 3-4 IVD were punctured with an 18-gauge needle at a depth of $5 \mathrm{~mm}$ and the incision was closed. L5-6 disc was used as a blank control. After the operation, rabbits were individually housed with free access to food and water.

\section{Injection of DD-SIS microparticles}

DD-SIS were collected and suspended in sterile normal saline at a concentration of $50 \mathrm{mg} / \mathrm{mL}$. Injections were performed one month after the IVD puncture. Rabbits ( 8 rabbits, all male, 2 months of age, weighted 2.2-2.8 kg, mean: $2.64 \mathrm{~kg}$ ) were anesthetized with an intravenous injection of $15 \mathrm{mg} / \mathrm{kg}$ pentobarbital sodium, and spines were exposed using a lateral retroperitoneal approach on the other side. Using a 28-g needle, the IVD of L4-5 and L3-4 were injected with DD-SIS suspension $(50 \mathrm{~mL})$ and normal saline, respectively. The small needle size was selected to avoid promoting further degeneration. After surgery, rabbits were individually housed with free access to food and water.

\section{MRI and X-ray evaluation}

Lumbar MRI was performed before the operation and at $0,1,2$ and 3 months postoperatively, with a GE Sigma CV/I (1.5T) using a knee-joint surface coil. Sagittal T2-weighted images were obtained using repetition/ echo times of $2500 / 120 \mathrm{~ms}$, bandwidth of $31.25 \mathrm{~Hz} / \mathrm{Px}$ and echo train length of 17, whereas T1-weighted used minifull $/ 550 \mathrm{~ms}, 31.25 \mathrm{~Hz} / \mathrm{Px}$, and echo train length of 2 . A central sagittal image of the lumbar spine was selected as a locating image for the next T2 FSE cross-sectional scans at $3000 / 102 \mathrm{~ms}$ and $31.25 \mathrm{~Hz} / \mathrm{Px}$ and echo train length of 17. The matrix size for each image was the same (matrix size, $384 \times 256$; field of view, $20 \times 20 \mathrm{~mm}$; slice thickness, $2 \mathrm{~mm}$; interslice gap, $0.2 \mathrm{~mm}$ ). Image J software 1.46 (National Institutes of Health, http://rsbweb. nih.gov/ij/download.html) was used to measure signal intensity of IVD of L3-4, L4-5, and L5-6 on T2-weighted central images. The signal intensity of cerebrospinal fluid was used as contrast. IVD height was also measured using X-ray analysis, calculated as the disc height index (DHI), which indicates the average height of the anterior, middle and posterior disc height. The change in DHI was calculated using a DHI of 0 month as contrast.

\section{Statistical analysis}

Quantitative data are expressed as mean \pm standard deviation (STD). Homogeneity test of variance were performed by Levene test, then analysis of variance (ANOVA) was used to compare mean data among groups, with differences between groups analyzed using the least significant difference (LSD) post-hoc analysis. Statistical analyses were performed using SPSS 16.0 (SPSS Inc., Chicago, IL, USA). $P<0.05$ was considered significant. 


\section{CONCLUSIONS}

In summary, we prepared SIS particles by decellularization, modified the SIS via NPC repopulation to make it suitable for IVD regeneration, and decellularized the material again before injection into a rabbit IDD model. The effect of inhibiting intervertebral disc degeneration progression was confirmed in vivo. This study showed that a xenogeneic decellularized SIS scaffold has potential therapeutic value for treating intervertebral disc degeneration.

\section{CONFLICTS OF INTEREST}

The authors declare no conflicts of interest.

\section{GRANT SUPPORT}

This work was supported by the National Natural Science Fund of China (81171739, 31270997 and 81672208), and Zhejiang Xinmiao Talents Program (grant number: 2016R413035)

\section{REFERENCES}

1. Nachemson AL, Wadell GI. NA. Epidemiology of neck and low back pain. In: Nachemson A L, E J, editors. neck and low back pain: the scientific evidence of causes, diagnosis and treatment. Philadelphia: Lippincott, Williams and Wilkins. 2000. p. 165-88.

2. Zheng CJ, Chen J. Disc degeneration implies low back pain. Theoretical biology \& medical modelling. 2015; 12:24.

3. Zhang YG, Guo TM, Guo X, Wu SX. Clinical diagnosis for discogenic low back pain. International journal of biological sciences. 2009; 5:647-58.

4. Silva-Correia J, Correia SI, Oliveira JM, Reis RL. Tissue engineering strategies applied in the regeneration of the human intervertebral disk. Biotechnology advances. 2013; 31:1514-31.

5. Crapo PM, Gilbert TW, Badylak SF. An overview of tissue and whole organ decellularization processes. Biomaterials. 2011; 32:3233-43.

6. Mercuri JJ, Gill SS, Simionescu DT. Novel tissue-derived biomimetic scaffold for regenerating the human nucleus pulposus. Journal of biomedical materials research Part A. 2011; 96:422-35.

7. Mercuri JJ, Patnaik S, Dion G, Gill SS, Liao J, Simionescu DT. Regenerative potential of decellularized porcine nucleus pulposus hydrogel scaffolds: stem cell differentiation, matrix remodeling, and biocompatibility studies. Tissue engineering Part A. 2013;19:952-66.

8. Xu H, Xu B, Yang Q, Li X, Ma X, Xia Q, Zhang Y, Zhang $\mathrm{C}, \mathrm{Wu} \mathrm{Y}$, Zhang Y. Comparison of decellularization protocols for preparing a decellularized porcine annulus fibrosus scaffold. PloS one. 2014; 9:e86723.
9. Chan LK, Leung VY, Tam V, Lu WW, Sze KY, Cheung KM. Decellularized bovine intervertebral disc as a natural scaffold for xenogenic cell studies. Acta biomaterialia. 2013; 9:5262-72.

10. Yuan M, Yeung CW, Li YY, Diao H, Cheung KM, Chan D, Cheah K, Chan PB. Effects of nucleus pulposus cell-derived acellular matrix on the differentiation of mesenchymal stem cells. Biomaterials. 2013; 34:3948-61.

11. Lin X, Fang X, Wang Q, Hu Z, Chen K, Shan Z, Chen S, Wang J, Mo J, Ma J, Xu W, Qin A, Fan S. Decellularized allogeneic intervertebral disc: natural biomaterials for regenerating disc degeneration. Oncotarget. 2016; 7:12121-36. doi: 10.18632/oncotarget.7735.

12. Gilchrist CL, Darling EM, Chen J, Setton LA. Extracellular matrix ligand and stiffness modulate immature nucleus pulposus cell-cell interactions. PloS one. 2011; 6:e27170.

13. Gilchrist CL, Francisco AT, Plopper GE, Chen J, Setton LA. Nucleus pulposus cell-matrix interactions with laminins. European cells \& materials. 2011;21:523-32.

14. Rodrigues-Pinto R, Richardson SM, Hoyland JA. An understanding of intervertebral disc development, maturation and cell phenotype provides clues to direct cell-based tissue regeneration therapies for disc degeneration. European spine journal. 2014; 23:1803-14.

15. Matsumoto T, Holmes RH, Burdick CO, Heisterkamp CA 3rd, O'Connell TJ Jr. Replacement of large veins with free inverted segments of small bowel: autografts of submucosal membrane in dogs and clinical use. Annals of surgery. 1966; 164:845-8.

16. Lawler MR Jr, Foster JH, Scott HW Jr. Evaluation of canine intestinal submucosa as a vascular substitute. American journal of surgery. 1971; 122:517-9.

17. Pu LL. Small intestinal submucosa (Surgisis) as a bioactive prosthetic material for repair of abdominal wall fascial defect. Plastic and reconstructive surgery. 2005; 115:2127-31.

18. McDevitt CA, Wildey GM, Cutrone RM. Transforming growth factor-betal in a sterilized tissue derived from the pig small intestine submucosa. Journal of biomedical materials research Part A. 2003; 67:637-40.

19. Ledet EH, Carl AL, DiRisio DJ, Tymeson MP, Andersen LB, Sheehan CE, Kallakury B, Slivka M, Serhan H. A pilot study to evaluate the effectiveness of small intestinal submucosa used to repair spinal ligaments in the goat. The spine journal. 2002; 2:188-96.

20. Liang R, Woo SL, Takakura $\mathrm{Y}$, Moon DK, Jia F, Abramowitch SD. Long-term effects of porcine small intestine submucosa on the healing of medial collateral ligament: a functional tissue engineering study. Journal of orthopaedic research. 2006; 24:811-9.

21. Musahl V, Abramowitch SD, Gilbert TW, Tsuda E, Wang JH, Badylak SF, Woo SL. The use of porcine small intestinal submucosa to enhance the healing of the medial collateral ligament--a functional tissue engineering study in rabbits. Journal of orthopaedic research. 2004; 22:214-20.

22. Bertone AL, Goin S, Kamei SJ, Mattoon JS, Litsky AS, Weisbrode SE, Clarke RB, Plouhar PL, Kaeding CC. 
Metacarpophalangeal collateral ligament reconstruction using small intestinal submucosa in an equine model. Journal of biomedical materials research Part A. 2008; 84:219-29.

23. Badylak SF, Tullius R, Kokini K, Shelbourne KD, Klootwyk T, Voytik SL, Kraine MR, Simmons C. The use of xenogeneic small intestinal submucosa as a biomaterial for Achilles tendon repair in a dog model. Journal of biomedical materials research. 1995; 29:977-85.

24. Valentin JE, Turner NJ, Gilbert TW, Badylak SF. Functional skeletal muscle formation with a biologic scaffold. Biomaterials. 2010; 31:7475-84.

25. Puglisi F, Capuano P, Iambrenghi OC, Armenise N, Carlucci F, Memeo M, Memeo R, Martines G. Laparoscopic repair of Morgagni hernia in an adult: use of a porcine small intestine submucosa biocompatible prosthesis. Chirurgia italiana. 2009; 61:351-6.

26. Badylak SF, Hoppo T, Nieponice A, Gilbert TW, Davison JM, Jobe BA. Esophageal preservation in five male patients after endoscopic inner-layer circumferential resection in the setting of superficial cancer: a regenerative medicine approach with a biologic scaffold. Tissue engineering Part A. 2011; 17:1643-50.

27. Mostow EN, Haraway GD, Dalsing M, Hodde JP, King D. Effectiveness of an extracellular matrix graft (OASIS Wound Matrix) in the treatment of chronic leg ulcers: a randomized clinical trial. Journal of vascular surgery. 2005; 41:837-43.

28. Rosen M, Roselli EE, Faber C, Ratliff NB, Ponsky JL, Smedira NG. Small intestinal submucosa intracardiac patch: an experimental study. Surgical innovation. 2005; 12:227-31.

29. Ashley RA, Roth CC, Palmer BW, Kibar Y, Routh JC, Fung KM, Frimberger D, Lin HK, Kropp BP. Regional variations in small intestinal submucosa evoke differences in inflammation with subsequent impact on tissue regeneration in the rat bladder augmentation model. BJU international. 2010; 105:1462-8.

30. Hiles MC, Badylak SF, Geddes LA, Kokini K, Morff RJ. Porosity of porcine small-intestinal submucosa for use as a vascular graft. Journal of biomedical materials research. 1993; 27:139-44.

31. Yang B, Zhou L, Sun Z, Yang R, Chen Y, Dai Y. In vitro evaluation of the bioactive factors preserved in porcine small intestinal submucosa through cellular biological approaches. Journal of biomedical materials research Part A. 2010; 93:1100-9.

32. Li F, Li W, Johnson S, Ingram D, Yoder M, Badylak S. Low-molecular-weight peptides derived from extracellular matrix as chemoattractants for primary endothelial cells. Endothelium. 2004; 11:199-206.

33. Du XF, Kwon SK, Song JJ, Cho CG, Park SW. Tracheal reconstruction by mesenchymal stem cells with small intestine submucosa in rabbits. International journal of pediatric otorhinolaryngology. 2012; 76:345-51.
34. Keane TJ, Londono R, Turner NJ, Badylak SF. Consequences of ineffective decellularization of biologic scaffolds on the host response. Biomaterials. 2012; 33:1771-81.

35. Nord A, Haugejorden O. [Two-year trial of the fluoridecontaining varnishes Duraphat and Carex]. Den Norske tannlaegeforenings tidende. 1991; 101:46-9.

36. Hoffman BE, Newman-Tarr TM, Gibbard A, Wang S, Hanning C, Pratta MA, Boyle RJ, Kumar S, Majumdar MK. Development and characterization of a human articular cartilage-derived chondrocyte cell line that retains chondrocyte phenotype. Journal of cellular physiology. 2010; 222:695-702.

37. Lefebvre V, Huang W, Harley VR, Goodfellow PN, de Crombrugghe B. SOX9 is a potent activator of the chondrocyte-specific enhancer of the pro alpha1(II) collagen gene. Molecular and cellular biology. 1997; 17:2336-46.

38. Bell DM, Leung KK, Wheatley SC, Ng LJ, Zhou S, Ling KW, Sham MH, Koopman P, Tam PP, Cheah KS. SOX9 directly regulates the type-II collagen gene. Nature genetics. 1997; 16:174-8.

39. Lau TT, Wang C, Png SW, Su K, Wang DA. Genipincrosslinked microcarriers mediating hepatocellular aggregates formation and functionalities. Journal of biomedical materials research Part A. 2011; 96:204-11.

40. Malda J, Frondoza CG. Microcarriers in the engineering of cartilage and bone. Trends in biotechnology. 2006; 24:299-304.

41. Takegami K, An HS, Kumano F, Chiba K, Thonar EJ, Singh K, Masuda K. Osteogenic protein-1 is most effective in stimulating nucleus pulposus and annulus fibrosus cells to repair their matrix after chondroitinase $\mathrm{ABC}$-induced in vitro chemonucleolysis. The spine journal. 2005; 5:231-8.

42. Stoyanov JV, Gantenbein-Ritter B, Bertolo A, Aebli N, Baur M, Alini M, Grad S. Role of hypoxia and growth and differentiation factor-5 on differentiation of human mesenchymal stem cells towards intervertebral nucleus pulposus-like cells. European cells \& materials. 2011; 21:533-47.

43. Tim Yoon S, Su Kim K, Li J, Soo Park J, Akamaru T, Elmer WA, Hutton WC. The effect of bone morphogenetic protein-2 on rat intervertebral disc cells in vitro. Spine. 2003; 28:1773-80.

44. Keorochana G, Johnson JS, Taghavi CE, Liao JC, Lee KB, Yoo JH, Ngo SS, Wang JC. The effect of needle size inducing degeneration in the rat caudal disc: evaluation using radiograph, magnetic resonance imaging, histology, and immunohistochemistry. The spine journal. 2010; 10:1014-23.

45. Kim MS, Lee MS, Song IB, Lee SJ, Lee HB, Khang G, Lee IW. Preparation of sponge using porcine small intesinal submucosa and their applications as a scaffold and a wound dressing. Advances in experimental medicine and biology. 2006;585:209-22.

46. Pei M, He F. Extracellular matrix deposited by synoviumderived stem cells delays replicative senescent chondrocyte 
dedifferentiation and enhances redifferentiation. Journal of cellular physiology. 2012; 227:2163-74.

47. Chen S, Huang Y, Zhou ZJ, Hu ZJ, Wang JY, Xu WB, Fang XQ, Fan SW. Upregulation of tumor necrosis factor alpha and ADAMTS-5, but not ADAMTS-4, in human intervertebral cartilage endplate with modic changes. Spine. 2014; 39:E817-25.
48. Sobajima S, Kompel JF, Kim JS, Wallach CJ, Robertson DD, Vogt MT, Kang JD, Gilbertson LG. A slowly progressive and reproducible animal model of intervertebral disc degeneration characterized by MRI, X-ray, and histology. Spine. 2005; 30:15-24. 